This item was submitted to Loughborough's Research Repository by the author.

Items in Figshare are protected by copyright, with all rights reserved, unless otherwise indicated.

\title{
Efficient LTE/WiFi coexistence in unlicensed spectrum using virtual network entity
}

PLEASE CITE THE PUBLISHED VERSION

https://doi.org/10.1109/GLOCOM.2017.8254978

PUBLISHER

(C) IEEE

VERSION

AM (Accepted Manuscript)

LICENCE

CC BY-NC-ND 4.0

\section{REPOSITORY RECORD}

Shoaei, Atoosa Dalili, Mahsa Derakhshani, Tho Le-Ngoc, and Mohamed Salem. 2019. "Efficient Lte/wifi Coexistence in Unlicensed Spectrum Using Virtual Network Entity". figshare.

https://hdl.handle.net/2134/26473. 


\title{
Efficient LTE/WiFi Coexistence in Unlicensed Spectrum using Virtual Network Entity
}

\author{
Atoosa Dalili Shoaei*, Mahsa Derakhshani ${ }^{\dagger}$, Tho Le-Ngoc*, Mohamed Salem ${ }^{\ddagger}$ \\ *Department of Electrical \& Computer Engineering, McGill University, Montreal, QC, Canada \\ $\dagger$ Wolfson School of Mechanical, Electrical and Manufacturing Engineering, Loughborough University, UK \\ $\ddagger$ Huawei Technologies Canada CO., LTD, ON, Canada
}

\begin{abstract}
Due to the increasing demand for mobile traffic, the unlicensed band operation for LTE is proposed by mobile operators. Although by using this approach higher capacity can be achieved for LTE, performance of other wireless technologies operating in this band such as WiFi can be degraded significantly. In order to enable efficient LTE/WiFi coexistence, we consider a coordinated structure via a virtual network entity. LTE users can transmit in the assigned time-slots, while WiFi users can compete with each other by using $p$-persistent CSMA in their exclusive time-share. In an unsaturated network, at each duty cycle, the TDMA scheduling for LTE users and $p$ values for WiFi users are updated to maximize the overall network throughput subject to a constraint on the minimum acceptable throughput for WiFi. The corresponding optimization problem is formulated and an iterative algorithm is developed to find the optimal solution using complementary geometric programming (CGP) and monomial approximations. The simulation results reveal the performance gains of the proposed algorithm in preserving the WiFi throughput requirement.
\end{abstract}

\section{INTRODUCTION}

LTE operation on unlicensed bands is considered by ThirdGeneration Partnership Project (3GPP) as a promising solution to meet the growing wireless data demand and to improve the spectrum efficiency. Although transmission across both unlicensed and licensed bands can provide a boost in LTE performance, such approach may cause a huge performance loss for $\mathrm{WiFi}$ that only uses unlicensed bands. The reason is that LTE networks use a schedule-based channel access, while WiFi employs a contention-based scheme, in which the user would randomly access the channel once it is detected idle. Therefore, in a coexistence scenario with LTE and WiFi sharing the same spectrum, starvation may happen for WiFi as the whole airtime may be occupied by LTE network [1]-[4].

In order to address this issue, two approaches have been so far proposed, LTE-Unlicensed (LTE-U) and licensed-assisted access (LAA). In LTE-U, which is developed by LTE-U Forum based on 3GPP Releases 10/11/12, a duty-cycle-based approach is used in which at each time-frame, LTE transmits over only a portion of a frame, leaving the rest of the time frame for WiFi. The main problem of this approach is that, since LTE performs no carrier sensing before transmission, on-going WiFi transmissions in the LTE subframe might be interrupted by LTE transmissions. This happens because there is no coordination among $\mathrm{WiFi}$ and LTE systems, thus $\mathrm{WiFi}$ users are unaware of LTE subframe. On the other hand, in LAA featured in 3GPP Release 13, the LTE base station (BS) is equipped with listen-before-talk mechanism, i.e., carrier sensing is performed before any transmission [5]. Although this approach may lead to better performance for WiFi compared to the LTE-U approach, the utilization still cannot reach to the optimal point due to the lack of coordination between LTE and WiFi. The analysis in [6] indicates that the LTE burst-transmission probability can be optimized to achieve the proportional fairness between LTE and WiFi.

In this paper, to enable efficient LTE/WiFi coexistence we consider a virtual network entity to coordinate the LTE and WiFi channel access [7]. This network entity facilitates separating LTE and WiFi transmissions in two different phases. LTE users can access the channel in the TDMA phase, while in the other phase, WiFi users can opportunistically transmit their packets using $p$-persistent CSMA. Moreover, this network entity can enable dynamic scheduling by assigning time-slots to LTE users and adjusting $p$ for WiFi users. This can improve the network throughput and preserve the WiFi throughput requirement. Using such approach, the unlicensed band can be used for both uplink and downlink transmissions.

In this work, assuming an unsaturated network for both LTE and $\mathrm{WiFi}$, the goal is to maximize the overall throughput over each frame, while the WiFi throughput does not fall below a target threshold. In other words, this scheme acts like a duty-cycle-based approach, where a period of a variable length time-frame is assigned exclusively to LTE, which cannot be used by WiFi users. The corresponding optimization problem is further formulated by complementary geometric programming and solved by an iterative algorithm.

The rest of this paper is organized as follows. We first introduce the system model in Section II. Section III presents the problem formulation and transformation of the optimization problem into CGP. Section IV presents the illustrative results and we provide some concluding remarks in Section V.

\section{System Model}

\section{A. Network Model and Frame Structure}

We consider an IEEE 802.11-based WLAN with $N_{\mathrm{W}}$ users, sharing the channel with an U-LTE network serving $N_{\mathrm{L}}$ users. In order to enable efficient coexistence between these two networks, we assume a virtual network entity to coordinate both the WiFi AP and U-LTE BS channel access using the following duty-cycle-based approach. At each duty cycle $t$, the time frame with a fixed duration of $T$, is divided into two 


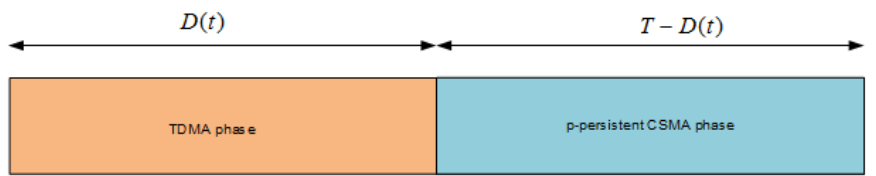

Fig. 1: Duty-cycle-based frame structure for WiFi and U-LTE coexistence

phases as shown in Fig 1. In the first phase with variable duration $D(t)<T$, LTE users can access the channel in the TDMA manner, while in the second phase with duration $T-D(t)$ WiFi users can opportunistically transmit their packets using $p$-persistent CSMA.

We assume that each user $n$ regardless of its network, has a queue with maximum length of $Q_{\max }$. At each duty cycle, with probability of $a_{n}$, a new packet is added to the queue of user $n$ if its length is smaller than $Q_{\max }$. Otherwise, the packet is discarded. Furthermore, we assume that the central controller is aware of packet arrival probabilities of users and it keeps a vector denoted by $\boldsymbol{V}(t)$, where $v_{n}(t)$ denotes the time that it has received the last packet from user $n$. Moreover, each time the user sends a packet, it piggybacks an extra bit (denoted by $\left.q_{n}(t)\right)$ telling if its queue is empty $\left(q_{n}(t)=0\right.$ ) or non-empty $\left(q_{n}(t)=1\right.$, i.e., it has packets backlogged in the queue to transmit). Therefore, at the duty cycle $t$, the controller updates $\theta_{n}(t)$, which indicates the probability that user $n$ has a non-empty queue at $t$, as

$$
\theta_{n}(t)= \begin{cases}1-\left(1-a_{n}\right)^{t-v_{n}(t)}, & \text { if } q_{n}\left(v_{n}(t)\right)=0 \\ 1 & \text { if } q_{n}\left(v_{n}(t)\right)=1\end{cases}
$$

\section{B. Throughput Analysis of p-persistent CSMA}

The WiFi system operates on the CSMA protocol. Using a $p$-persistent CSMA, a WiFi user with a packet to send will sense the channel. If the channel is sensed to be idle, the WiFi user $n_{w}$ will transmit the packet with probability $p_{n_{w}}$ at the beginning of the next backoff unit and defers with probability $1-p_{n_{w}}$. If the channel detected busy, the user waits until it becomes idle.

In the following, we derive the throughput of $p$-persistent CSMA protocol in an unsaturated network. The probability that channel is idle in a smallest possible backoff time duration, $\delta$, can be calculated as

$$
P_{\text {idle }}=\prod_{n_{w} \in \mathcal{N}_{\mathrm{W}}}\left(1-\theta_{n_{w}} p_{n_{w}}\right),
$$

where $\theta_{n_{w}} p_{n_{w}}$ represents the transmission probability of user $n_{w}$. Furthermore, we calculate the probability of successful transmission which happens if only one user transmits on the channel. This probability for transmission initiated by user $n_{w}$ (denoted by $P_{\text {succ }}^{n_{w}}$ ) can be obtained as

$$
P_{\text {succ }}^{n_{w}}=\theta_{n_{w}} p_{n_{w}} \prod_{n_{w}^{\prime} \in \mathcal{N}_{\mathrm{w}}, n_{w}^{\prime} \neq n_{w}}\left(1-\theta_{n_{w}^{\prime}} p_{n_{w}^{\prime}}\right) .
$$

As introduced in [8], we define the normalized throughput of user $n_{w}$ (denoted by $\rho_{n_{w}}$ ) as the fraction of time the channel is used for its successful transmission. Accordingly, $\rho_{n_{w}}$ can be written as

$$
\rho_{n_{w}}=\frac{P_{\text {succ }}^{n_{w}} T_{s}}{P_{\text {idle }} \delta+\left(1-P_{\text {idle }}\right) T_{s}},
$$

where $\delta$ is the backoff duration and $T_{s}$ is the duration of a fixed-size packet transmission time-slot, including the data transmission time, guard-time, and signaling overheads. Since signaling and guard-time are relatively small (in the order of $\mu \mathrm{s}$ ) compared with data transmission (in the order of $\mathrm{ms}$ ), we approximately assume that both collided and successful transmissions are of the same size (i.e., $T_{s}$ ). Consequently, the denominator in (4) represents the expected length of a general time-slot.

We can simplify (4) by introducing a new variable, i.e.,

$$
y_{n_{w}}=\frac{\theta_{n_{w}} p_{n_{w}}}{1-\theta_{n_{w}} p_{n_{w}}} .
$$

To this end, first, we rewrite $P_{\text {idle }}$ and $P_{\text {succ }}^{n_{w}}$ in terms of $y_{n_{w}}$ as

$$
\begin{aligned}
P_{\text {idle }} & =\frac{1}{\prod_{n_{w} \in \mathcal{N}_{w}}\left(1+y_{n_{w}}\right)} \\
P_{\text {succ }}^{n_{w}} & =\frac{y_{n_{w}}}{\prod_{n_{w} \in \mathcal{N}_{w}}\left(1+y_{n_{w}}\right)}=y_{n_{w}} P_{\text {idle }} .
\end{aligned}
$$

Then, we obtain $\rho_{n_{w}}$ in terms of $y_{n_{w}}$ as

$$
\rho_{n_{w}}=\frac{y_{n_{w}}}{\prod_{n_{w} \in \mathcal{N}_{w}}\left(1+y_{n_{w}}\right)-t^{\prime}},
$$

where $t^{\prime}=\frac{T_{s}-\delta}{T_{s}}$.

\section{HYBRID TDMA-CSMA SCHEDULING VIA CGP FOR WIFI AND LTE COEXISTENCE}

To date, unlicensed spectrum is used by wireless technologies such as WiFi, bluetooth, and radar. However as cellular networks are facing capacity issues, operating LTE in the unlicensed spectrum (U-LTE) has been proposed by the 3GPP standardization group. Although this approach may attract interest from LTE side, however, it may cause interference for WiFi users [7]. In order to enable efficient coexistence between LTE and WiFi across the unlicensed spectrum, virtualized network entities can be used, where users from different wireless technologies form different slices: LTE slice and WiFi slice. We consider the scenario that each network only serves its own users, i.e. the user has one active access network connection (e.g., either LTE or WiFi). Therefore, LTE users can only transmit in TDMA time-slot, while WiFi users compete with each other in the CSMA time-slot. In other words, each network uses the current deployed MAC protocol with negligible modification.

\section{A. Problem Formulation}

Let assume that the LTE BS and WiFi AP are connected to a virtual network entity. This virtualized architecture supports 
separation of the data plane from the control plane, which enables efficient centralized management of the radio access network (RAN).

To facilitate coexistence between WiFi and LTE and increase the spectral efficiency, the central controller dynamically divides each duty cycle between two slices, aiming to maximize the overall throughput, while maintaining the WiFi throughput not degraded significantly compared to the case in which the band is not shared with LTE. In particular, this problem can be formulated as follows,

$$
\begin{array}{ll}
\max _{\boldsymbol{X}, \boldsymbol{Y}} & S_{\mathrm{td}}(t)+S_{\mathrm{cs}}(t), \quad \text { subject to, } \\
\mathrm{C} 1: & S_{\mathrm{cs}}(t) \geq \eta .
\end{array}
$$

In this optimization problem, the objective function represents the total throughput of network in both TDMA and CSMA phases in the duty cycle $t$. It should be noted that in this setting, the throughput in the TDMA and CSMA time-slots represents the throughput of the U-LTE and WiFi systems, respectively. In addition, the constraint is to guarantee that the WiFi throughput does not fall below a required threshold (denoted by $\eta$ ).

Considering the throughput of each WiFi user in (8), the optimization problem in (9) can be expanded as

$$
\max _{\boldsymbol{X}, \boldsymbol{Y}} \sum_{n_{l} \in \mathcal{N}_{\mathrm{L}}} \theta_{n_{l}} x_{n_{l}}+\sum_{n_{w} \in \mathcal{N}_{\mathrm{W}}} \frac{y_{n_{w}}\left(T-T_{s} \sum_{n_{l} \in \mathcal{N}_{\mathrm{L}}} x_{n_{l}}\right)}{\prod_{n_{w} \in \mathcal{N}_{\mathrm{W}}}\left(1+y_{n_{w}}\right)-t^{\prime}}
$$

subject to:

$$
\mathrm{C} 1: \sum_{n_{w} \in \mathcal{N}_{\mathrm{W}}} \frac{y_{n_{w}}\left(T-T_{s} \sum_{n_{l} \in \mathcal{N}_{\mathrm{L}}} x_{n_{l}}\right)}{\prod_{n_{w} \in \mathcal{N}_{\mathrm{W}}}\left(1+y_{n_{w}}\right)-t^{\prime}} \geq \eta,
$$

where $Y$ is the vector defined in Equation (5) and $X=\left[x_{n_{l}}\right]$

is the vector indicating the time-slot allocation for LTE users in one duty cycle. In particular, $x_{n_{l}} \in\{0,1\}$, where $x_{n_{l}}=1$ if a time-slot is allocated to the LTE user $n_{l}$ in the TDMA timeslot and $x_{n_{l}}=0$ otherwise. Thus, the expected throughput associated with U-LTE users in the TDMA time-slot is $S_{\mathrm{td}}=$ $\sum_{n_{l} \in \mathcal{N}_{\mathrm{L}}} \theta_{n_{l}} x_{n_{l}}$. It should be noted that the second term in (10a) represents the entire WiFi network throughput, i.e., $S_{\mathrm{cs}}=$ $T_{\mathrm{cs}} \sum_{n_{w} \in \mathcal{N}_{\mathrm{W}}} \rho_{n_{w}}$, where $T_{\mathrm{cs}}$ denotes the duration of CSMA time-slot in a duty cycle.

Clearly, the optimization problem in (10) has a non-convex objective function and a non-convex constraint with the combination of continuous and binary variables, i.e., $y_{n_{w}}\left(y_{n_{w}} \geq 0\right)$ and $x_{n_{l}}\left(x_{n_{l}} \in\{0,1\}\right)$. Thus, (10) is a non-convex mixedinteger, NP-hard optimization problem. To solve this issue, we first relax the integer variable, i.e. $X$, to continuous one. Then, we aim to transform the problem to a form that can be solved in an efficient manner. In the following, we explain how this transformation can be done.

With a closer look, the optimization problem in (10) potentially looks like an extension of Geometric Programming (GP). A GP is an optimization problem of the form

$$
\begin{array}{ll}
\min _{\boldsymbol{x}} & f_{0}(\boldsymbol{x}) \\
\text { s.t. : } & f_{i}(\boldsymbol{x}) \leq 1, i=1,2, \ldots, I \\
& g_{j}(\boldsymbol{x})=1, j=1,2, \ldots, J,
\end{array}
$$

where $\boldsymbol{x}=\left[x_{1}, \ldots, x_{N}\right]$ is a non-negative vector of optimization variables, $g_{j}(\boldsymbol{x})=c_{i} \prod_{n=1}^{N} x_{n}^{b_{i, n}}$ for all $j$ are monomial functions, and $f_{i}(\boldsymbol{x})=\sum_{k=1}^{K_{j}} c_{j, k} \prod_{n=1}^{N} x_{n}^{b_{j, k, n}}$ are posynomial functions for $i=0, \ldots, I$, where the multiplicative constants are positive (i.e., $c_{i}, c_{j, k}>0$ ) and $b_{i, n}, b_{j, k, n} \in \boldsymbol{R}$.

The ultimate goal is to transform the optimization problem in (10) to a GP form. To this end, we first multiply the objective function by -1 to change the problem to a minimization problem. Then, since in GP the objective function should be positive, we add a sufficiently large constant $M$ to the objective function. Furthermore, we employ two auxiliary variables $T_{\mathrm{cs}}=T-T_{s} \sum_{n_{l} \in \mathcal{N}_{L}} x_{n_{l}}$ and $d=\prod_{n_{w} \in \mathcal{N}_{\mathrm{w}}}\left(1+y_{n_{w}}\right)-t^{\prime}$. By replacing of these auxiliary variables with their corresponding terms and applying transformations in the objective function, (10) becomes

$$
\min _{\boldsymbol{X}, \boldsymbol{Y}, T_{\mathrm{cs}}, d} M-\sum_{n_{l} \in \mathcal{N}_{\mathrm{L}}} \theta_{n_{l}} x_{n_{l}}-\sum_{n_{w} \in \mathcal{N}_{w}} T_{\mathrm{cs}} y_{n_{w}} d^{-1}
$$

subject to:

$$
\begin{aligned}
& \mathrm{C} 1: \sum_{n_{w} \in \mathcal{N}_{\mathrm{W}}} T_{\mathrm{cs}} y_{n_{w}} d^{-1} \geq \eta, \\
& \mathrm{C} 2: d=\prod_{n_{w} \in \mathcal{N}_{\mathrm{W}}}\left(1+y_{n_{w}}\right)-t^{\prime} \\
& \mathrm{C} 3: T_{\mathrm{cs}}=T-T_{s} \sum_{n_{l} \in \mathcal{N}_{L}} x_{n_{l}} .
\end{aligned}
$$

In (12), the objective function is not a posynomial because of the negative in the second term. Thus, we introduce and minimize a new auxiliary variable $x_{0}$ in addition to guaranteeing the constraint C4 in (13). Finally, we reach to the following optimization problem

$$
\begin{aligned}
& \min _{\boldsymbol{X}, \boldsymbol{Y}, T_{\mathrm{cs}}, d, x_{0}} x_{0}, \text { subject to: } \\
& \mathrm{C} 1: \frac{\eta}{\sum_{n_{w} \in \mathcal{N}_{\mathrm{W}}} T_{\mathrm{cs}} y_{n_{w}} d^{-1}} \leq 1 \\
& \mathrm{C} 2: \frac{\prod_{n_{w} \in \mathcal{N}_{\mathrm{W}}}\left(1+y_{n_{w}}\right)}{t^{\prime}+d}=1 \\
& \mathrm{C} 3: \frac{T}{T_{\mathrm{cs}}+T_{s} \sum_{n_{w} \in \mathcal{N}_{\mathrm{W}}} x_{n_{w}}}=1 \\
& \mathrm{C} 4: \frac{M}{x_{0}+\sum_{n_{l} \in \mathcal{N}_{\mathrm{L}}} \theta_{n_{l}} x_{n_{l}}+\sum_{n_{w} \in \mathcal{N}_{\mathrm{W}}} T_{\mathrm{cs}} y_{n_{w}} d^{-1}} \leq 1 .
\end{aligned}
$$

In this optimization problem, all upper-bound inequality constraints are in the form of a ratio between two posynomials and equality constraints are in the form of a ratio between a monomial and a posynomial. This problem belongs to the class of complementary geometric programming (CGP), which potentially looks like an extension of GP. In particular, a CGP 
can be presented as

$$
\begin{array}{cl}
\min _{\boldsymbol{x}} & P_{0}(\boldsymbol{x}) \\
\text { s.t. : } & P_{i}(\boldsymbol{x}) \leq 1, i=1, \ldots, I, \\
& Q_{j}(\boldsymbol{x})=1, j=1, \ldots, J,
\end{array}
$$

where $P_{0}(\boldsymbol{x})$ is a posynomial and $P_{i}(\boldsymbol{x})=\frac{p_{i}(\boldsymbol{x})}{p_{i}^{+}(\boldsymbol{x})}$ for all $i=1, \ldots, I$, in which $p_{i}(\boldsymbol{x})$ and $p_{i}^{+}(\boldsymbol{x})$ are posynomial functions. Moreover, $Q_{j}(\boldsymbol{x})=\frac{q_{j}(\boldsymbol{x})}{q_{j}^{+}(\boldsymbol{x})}$ for all $j$, in which $q_{j}(\boldsymbol{x})$ are monomial and $q_{j}^{+}(\boldsymbol{x})$ are posynomial functions.

\section{B. Algorithm}

In this section, we focus on the solution of the optimization problem and present a computationally efficient algorithm which provides a locally optimal solution. In this algorithm, by applying successive transformations, we can convert the CGP problem into a sequence of ordinary GP problems [9], [10].

By approximating the posynomials in the denominator of constraints in (14) with monomials, a complementary GP can be turned into a standard form of GP. The arithmetic-geometric mean inequality can be used to approximate a posynomial with a monomial. In the following, we describe the monomial approximation which is useful to transform the problem into GP. Let $p_{i}^{+}(\boldsymbol{x})=\sum_{k=1}^{K_{i}} m_{i, k}^{p}(\boldsymbol{x})$ and $q_{j}^{+}(\boldsymbol{x})=\sum_{k=1}^{K_{j}} m_{j, k}^{q}(\boldsymbol{x})$, where $m_{i, k}^{p}$ and $m_{j, k}^{q}$ are monomials. Using the arithmeticgeometric mean inequality, at iteration $l, p_{i}^{+}(\boldsymbol{x})$ and $q_{j}^{+}(\boldsymbol{x})$ can be approximated as

$$
\begin{aligned}
& \widetilde{p}_{i}^{+}(\boldsymbol{x}(l))=\prod_{k=1}^{K_{i}}\left(\frac{m_{i, k}^{p}(\boldsymbol{x}(l))}{\alpha_{i, k}(\boldsymbol{x}(l))}\right)^{\alpha_{i, k}(\boldsymbol{x}(l))}, \\
& \widetilde{q}_{j}^{+}(\boldsymbol{x}(l))=\prod_{k=1}^{K_{j}}\left(\frac{m_{j, k}^{q}(\boldsymbol{x}(l))}{\zeta_{j, k}(\boldsymbol{x}(l))}\right)^{\zeta_{j, k}(\boldsymbol{x}(l))},
\end{aligned}
$$

The parameters $\alpha_{i, k}(\boldsymbol{x}(l))$ and $\zeta_{j, k}(\boldsymbol{x}(l))$ can be computed as

$$
\begin{aligned}
& \alpha_{i, k}(\boldsymbol{x}(l))=\frac{m_{i, k}^{p}((\boldsymbol{x}(l-1))}{p_{i}^{+}((\boldsymbol{x}(l-1))}, \quad \forall i, k \\
& \zeta_{j, k}(\boldsymbol{x}(l))=\frac{m_{j, k}^{q}((\boldsymbol{x}(l-1))}{q_{j}^{+}((\boldsymbol{x}(l-1))}, \quad \forall k, j
\end{aligned}
$$

where $x(l-1)$ is the value of last-round solution of the optimization problem. It is proved that arithmetic-geometric mean approximation gives the best local monomial approximation for a posynomial function [11].

Using the approximations in (15) and (16), in each iteration, the optimization problem in (14) would be in the form of a standard GP problem. Consequently, the optimal solution can be achieved by iteratively applying monomial approximations and solving a series of GPs [9]. Using such iterative approach, we propose an algorithm to solve the problem in (13). The details of this approach are provided in Algorithm 1.

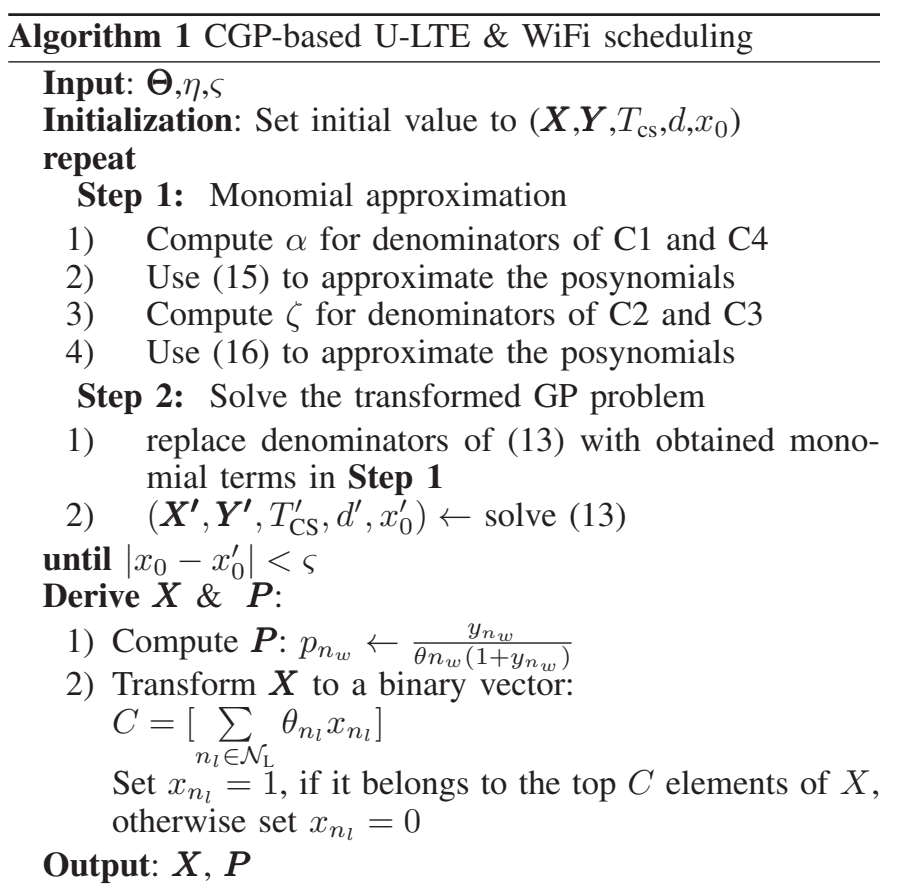

\section{Illustrative Results}

We consider a scenario with one LTE BS serving $N_{L}$ users and one WiFi AP serving $N_{W}$ users, both operating on the same channel and using the same packet transmission timeslot of $T_{s}$. Consequently, the frame $T$, and 2 phases $D(t)$, and $[T-D(t)]$ are represented as the integer numbers of timeslots. In the following, we set $T=19 T_{s}$ and present the considered scenarios for performance evaluation along with their results using MATLAB and CVX to derive the solutions of GP problems (13).

\section{A. Effects of Increasing $N_{L}$}

We first investigate how increasing the number of U-LTE users, $N_{L}$, can affect the WiFi throughput and the overall network throughput. For this scenario, we set the WiFi throughput threshold $\eta$ to 5 time-slots per frame and the number of WiFi users, $N_{\mathrm{W}}=12$. Moreover, the considered packet arrival probabilities are $A_{\mathrm{L}}=\left\{[0.8]_{4},[0.5]_{N_{\mathrm{L}}-4}\right\}$ and $\boldsymbol{A}_{\mathrm{W}}=\left\{[0.8]_{4},[0.5]_{8}\right\}$, i.e., the WiFi network serves 4 users with $a_{n}=0.8$ and 8 users with $a_{n}=0.5$. Similarly, U-LTE has 4 users with $a_{n}=0.8$ and the rest of its users are the ones with $a_{n}=0.5$.

As can be seen in Fig. 2, by increasing the number of ULTE users, i.e., $N_{\mathrm{L}}$, greater throughput can be achieved for both U-LTE system and the overall network. The reason is that more packets are generated, therefore with higher probability time-slots are allocated to the users who have packets for transmission. On the other hand, WiFi throughput decreases because greater throughput can be achieved by assigning more time-slots to the U-LTE system. However, due to the WiFi throughput constraint, WiFi throughput never falls below the targeted threshold. 


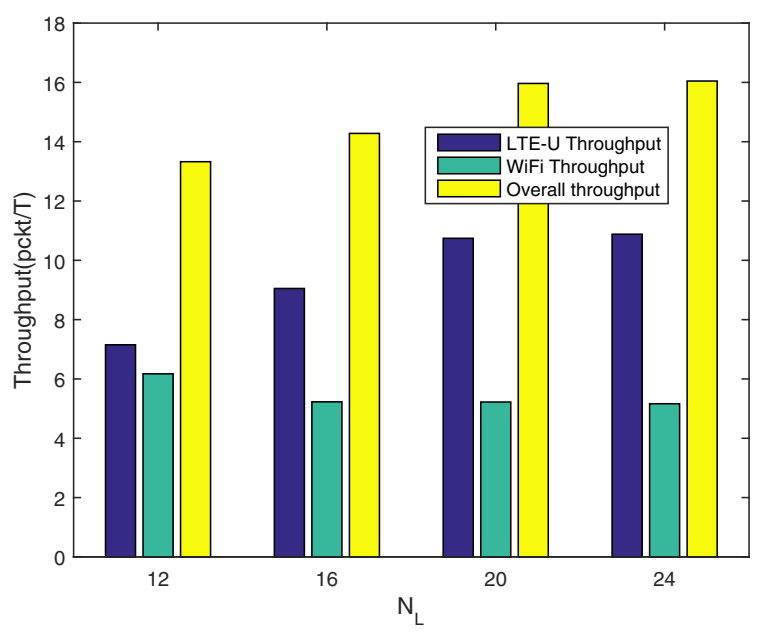

Fig. 2: Throughput vs $N_{\mathrm{L}}$

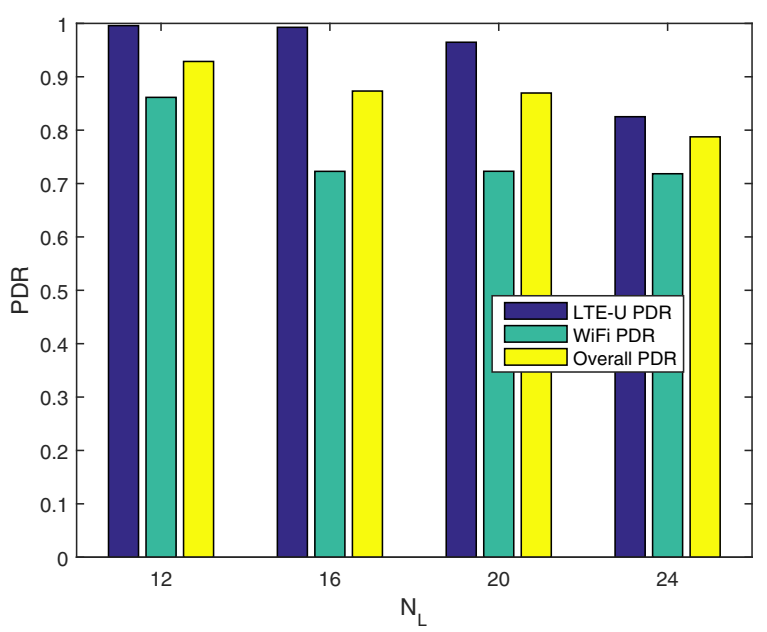

Fig. 3: PDR vs $N_{\mathrm{L}}$

Furthermore, the effects of increasing $N_{\mathrm{L}}$ on the packet delivery ratio (PDR) are demonstrated in Fig. 3. PRD is defined as the ratio of number of transmitted packets to the number of generated packets. As can be observed, for $N_{\mathrm{L}}=\{12,16\}$, U-LTE PDR is so close to 1 , however by increasing $N_{\mathrm{L}}$ it starts to drop, which means that U-LTE users' quality of service requirements would be affected. In order to avoid this situation, the number of U-LTE users should be controlled, otherwise U-LTE users will suffer from a performance degradation.

\section{B. Effects of Increasing $N_{W}$}

In another scenario, we have the results for increasing the number of Wifi users, with $A_{\mathrm{L}}=\left\{[0.8]_{4},[0.5]_{8}\right\}, \boldsymbol{A}_{\mathrm{W}}=$ $\left\{[0.8]_{4},[0.5]_{N_{w}-4}\right\}$ and $\eta=5$ time-slots per frame. In Fig. 4 , it is evident that both U-LTE and WiFi throughputs remain unchanged. However, as shown in Fig. 5, the WiFi PDR decreases due to the larger number of generated packets. The

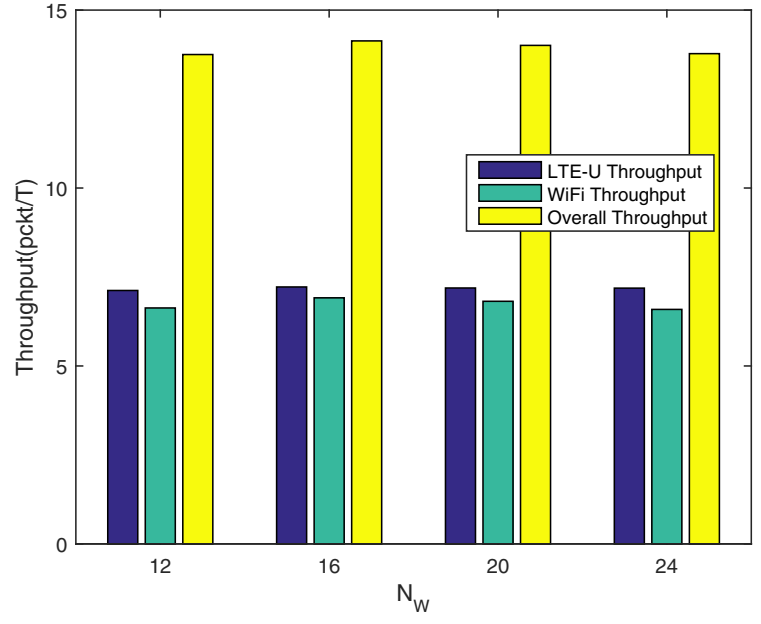

Fig. 4: Throughput vs $N_{W}$

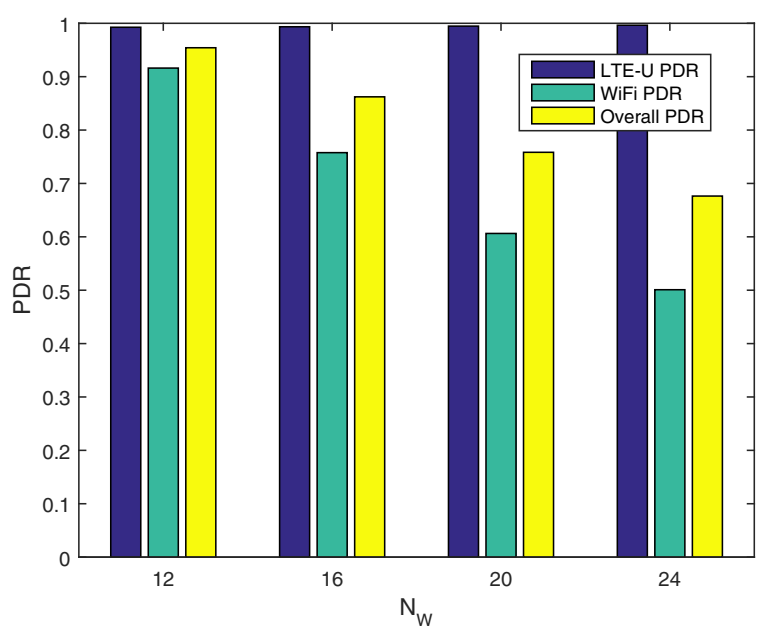

Fig. 5: PDR vs $N_{W}$

reason is that since higher throughput can be achieved by allocating time to U-LTE, increasing $N_{\mathrm{W}}$ does not lead to a larger share of time for WiFi. On the other hand, increasing $N_{\mathrm{W}}$ may lead to a larger number of collisions, therefore, to meet the WiFi throughput threshold, lower $p$ probabilities are assigned to the users.

\section{Effects of Increasing $\eta$}

We also obtain the results to show how the algorithm behaves when the threshold for average WiFi throughput, $\eta$, is incremented. For this scenario, we set the parameters as $\boldsymbol{A}_{\mathrm{L}}=\left\{[0.9]_{14}\right\}$ and $\boldsymbol{A}_{\mathrm{W}}=\left\{[1]_{14}\right\}$. As observed in Fig. 6, by increasing $\eta$, the total network throughput drops. The reason is that, in this scenario although higher total throughput can be achieved by assigning more time-slots to the U-LTE, WiFi throughput constraint must be satisfied as well.

Furthermore, we obtain the results for PDR shown in Fig. 7. Clearly, by increasing $\eta$, LTE PDR degrades, since less airtime 


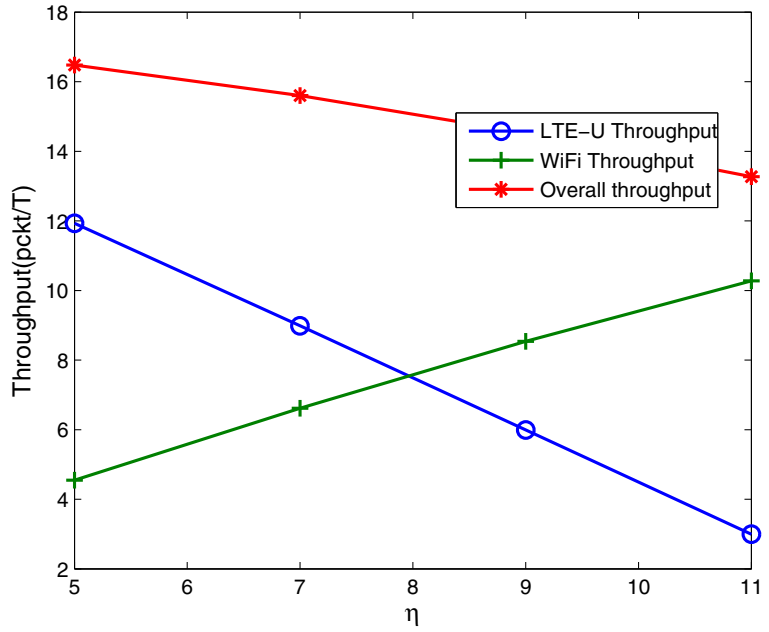

Fig. 6: Throughput vs $\eta$

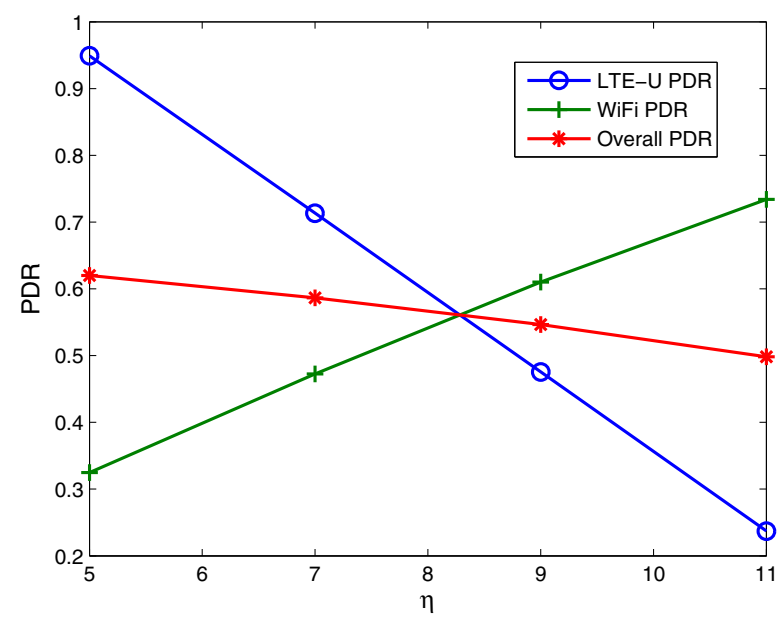

Fig. 7: PDR vs $\eta$

is allocated to LTE. On the other hand, WiFi PDR increases because due to the larger allocated airtime, more number of packets are transmitted. However, as WiFi PDR increment is less than LTE PDR decrement, the overall PDR drops down by increasing $\eta$.

\section{CONClusion}

In order to satisfy the increasing demand for mobile traffic, LTE operation over unlicensed bands has been proposed. In this paper, we consider the scenario that both LTE and WiFi systems share the same unlicensed band. In such a setting, the main challenge for U-LTE deployment is that the performance of WiFi system should not degrade significantly. In order to address this issue, we consider a coordinated approach via a virtual network entity. This entity manages the channel access between these two systems to improve the overall spectrum efficiency, while the WiFi performance does not fall below a certain level. In order to reach this goal, a duty-cyclebased approach is used, in which the time is divided into duty cycles and the exclusive share of each system is dynamically optimized by the network entity. It is shown that the developed algorithm can ensure a minimum level throughput requirement for WiFi while maximizing the total throughput.

\section{ACKNOWLEDGMENT}

This work is supported in part by an NSERC CRD Grant with Huawei Technologies Canada CO., LTD.

\section{REFERENCES}

[1] F. Chaves, A. Cavalcante, E. Almeida, F. Abinader Jr, R. Vieira, S. Choudhury, and K. Doppler, "LTE/Wi-Fi coexistence: Challenges and mechanisms," XXXI SIMPOSIO BRASILEIRO DE TELECOMUNICACOES, 2013.

[2] F. M. Abinader, E. P. Almeida, F. S. Chaves, A. M. Cavalcante, R. D. Vieira, R. C. Paiva, A. M. Sobrinho, S. Choudhury, E. Tuomaala, $\mathrm{K}$. Doppler et al., "Enabling the coexistence of LTE and Wi-Fi in unlicensed bands," IEEE Commun. Mag., vol. 52, no. 11, pp. 54-61, 2014.

[3] B. Chen, J. Chen, Y. Gao, and J. Zhang, "Coexistence of LTE-LAA and Wi-Fi on $5 \mathrm{GHz}$ with corresponding deployment scenarios: A survey," IEEE Commun. Surveys Tuts., vol. PP.

[4] A. Mukherjee, J.-F. Cheng, S. Falahati, H. Koorapaty, R. Karaki, L. Falconetti, D. Larsson et al., "Licensed-assisted access LTE: coexistence with IEEE 802.11 and the evolution toward 5G," IEEE Commun. Mag., vol. 54, no. 6, pp. 50-57, 2016.

[5] 3GPP Study Item RP-141397, "Study on licensed-assisted access using LTE," Tech. Rep., 2014.

[6] C. Cano and D. J. Leith, "Coexistence of WiFi and LTE in unlicensed bands: A proportional fair allocation scheme," in IEEE Intl. Conf. on Commun. Workshop (ICCW). IEEE, 2015, pp. 2288-2293.

[7] A. Al-Dulaimi, S. Al-Rubaye, Q. Ni, and E. Sousa, "5G communications race: pursuit of more capacity triggers LTE in unlicensed band," IEEE Trans. Veh. Technol., vol. 10, no. 1, pp. 43-51, 2015.

[8] G. Bianchi, "Performance analysis of the IEEE 802.11 distributed coordination function," IEEE J. Sel. Areas Commun., vol. 18, no. 3, pp. 535-547, Mar. 2000.

[9] G. Xu, "Global optimization of signomial geometric programming problems," European Journal of Operational Research, vol. 233, no. 3, pp. 500-510, 2014.

[10] M. Derakhshani, X. Wang, T. Le-Ngoc, and A. Leon-Garcia, "Airtime usage control in virtualized multi-cell 802.11 networks," in IEEE Globecom Workshops (GC Wkshps), Dec. 2015, pp. 1-6.

[11] S. Boyd and L. Vandenberghe, Convex optimization. Cambridge university press, 2004. 